\title{
H2A.Z acetylation and transcription: ready, steady, go!
}

\author{
"...studying the incorporation of H2A.Z as a whole without \\ considering the PTMs, is insufficient to understand the mechanism \\ of action of this histone variant."
}

First draft submitted: 19 February 2016; Accepted for publication: 23 February 2016; Published online: 18 April 2016

Keywords: acetylation $\bullet \mathrm{H} 2 \mathrm{~A} . \mathrm{Z} \bullet \mathrm{PTMs} \bullet$ transcription

In the cell nucleus, genomic DNA is packaged into chromatin, which consists of basic repeat units, called nucleosomes, which contain approximately 146 bp of DNA wrapped around an octamer of core histones (two molecules of each histone $\mathrm{H} 2 \mathrm{~A}, \mathrm{H} 2 \mathrm{~B}, \mathrm{H} 3$ and $\mathrm{H} 4$ ). The compaction of chromatin is a highly regulated and dynamic process and restricts the access of cellular machinery to DNA. Chemical modifications of the core histone tails and incorporation of histone variants act to modulate the chromatin structure, modifying the histone-DNA and histone-histone interactions. H2A.Z is an evolutionary conserved histone variant of the canonical H2A family, which shares approximately $60 \%$ amino-acid sequence. H2A.Z is essential for viability in many organisms, including mammals suggesting a unique and important role [1]. In fact H2A.Z has been implicated in many diverse and potentially contrasting functions such as gene transcription, nucleosome turnover, DNA repair, heterochromatin boundaries, chromosome segregation, progression through the cell cycle, suppression of antisense RNAs, embryonic stem cell (ESC) differentiation and antagonizing DNA methylation [1]. In particular several reports highlight potential conflicting roles of H2A.Z nucleosome occupancy in the regulation of gene activity, where H2A.Z has been implicated in active, poised or inactive gene expression [2-4]. A key question, therefore, is how can this variant have such a wide variety of functions, and potentially perform opposite roles?
Over the last 15 years, H2A.Z potential contradictory roles in gene transcription have been widely studied and it is now clear that there are multiple factors that affect H2A.Z repressive/poise/active roles [5]. One of these factors is H2A.Z posttranslation modification (PTM) (reviewed in [6]). H2A.Z lysines can be either acetylated (K4, K7 and K11) [7,8] or methylated (K4 and K7) [9] at the N-terminal. In addition, three specific lysines (K120, K121 and K125) at the C-terminal can be monoubiquitinated [10]. Methylation of lysines 4 and 7 are required for ESC self-renewal and has been associated with negative regulation of gene expression [9]. Monoubiquitilated H2A.Z is found at facultative heterochromatin and is associated with repressed transcription [10,11], while acetylation is a mark of active chromatin [7,12-14] and confers nucleosome destabilization and an open conformation [8]. Interestingly, comodification of H2A.Z with both acetylation and monoubiquitination, is enriched at bivalent chromatin in ESCs [15].

Despite the reported existence and potential confounding functions of these PTMs, the scientific community primarily studies H2A.Z and its role in transcription blinded to PTM state of this histone variant, as the commonly used H2A.Z antibody in chromatin immunoprecipitation studies is agnostic to PTMs. In this editorial piece, we will summarize the scientific evidence supporting the thesis that PTMs, in particular
Epigenomics

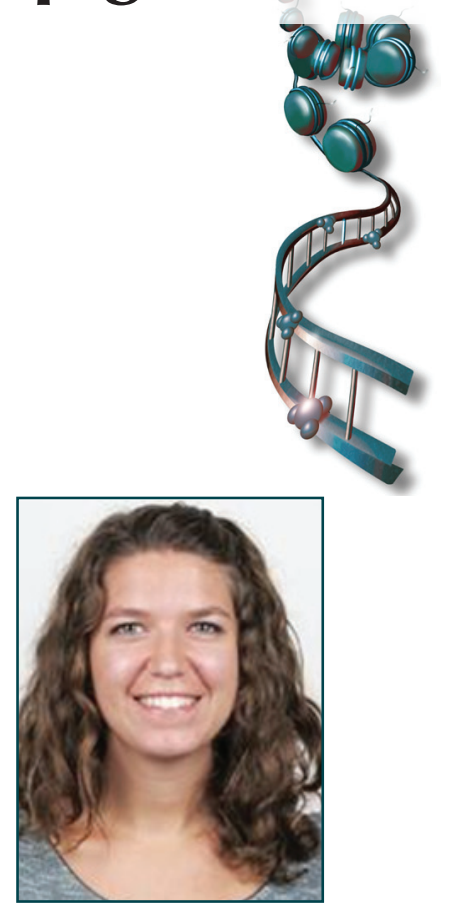

Yolanda Colino-Sanguino

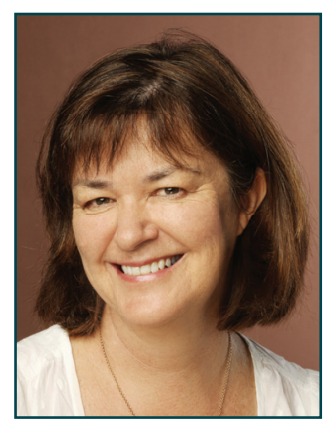

Susan J Clark ${ }^{\ddagger}$ *

Author for correspondence: s.clark@garvan.org.au

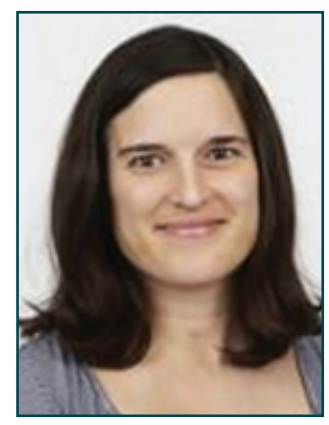

Fatima Valdes-Mora ${ }^{\ddagger}$ ${ }^{\ddagger}$ Authors contributed equally For a full list of affiliations, please see page 586

Future 
acetylation of H2A.Z (H2A.Zac), and not unmodified H2A.Z, are correlated and functionally involved with active gene transcription.

\section{"Even though it is now clear that H2A.Zac is a mark of active transcription, there are only a few studies reporting the functional link and phenotypic consequences of H2A.Z acetylation in the context of gene transcription."}

Acetylation is the best characterized modification of H2A.Z and plays a key role in transcriptional activation. In yeast, H2A.Z acetylation levels correlate with global gene activity $[7]$, and it is required for galactosedependent gene induction [12]. Similarly in chicken cells, the hyperacetylated form of H2A.Z is enriched at the 5' end of active genes but is absent from inactive genes [13]. In humans, there are a number of studies that associate gene expression with H2A.Zac, with implications in cell differentiation $[15,16]$ and cancer $[14,17-19]$. In fact, we have previously reported that $\mathrm{H} 2 \mathrm{~A}$. Zac is present at promoters of highly expressed genes in normal and prostate cancer cell lines. Moreover, deregulation of gene expression in cancer is associated with H2A.Zac/H2A.Z remodeling, where silent genes become active by lose of underacetylated H2A.Z and gain of acetylated H2A.Z nucleosomes at gene promoters [14]. In accordance with our previous study, others have further reported the enrichment of promoter-associated H2A.Zac nucleosomes and active gene transcription. For example, in p53 negative breast cancer cells, depletion of H2A.Z is not enough to trigger p21 transcription but needs to be accompanied by an increase in promoter H2A.Zac levels [19]. In addition, the expression of the oncogene cyclin D1 requires eviction of non-acetylated H2A.Z and acetylation of the remaining H2A.Z-containing nucleosomes at the promoter and enhancer in estrogen receptor (ER) positive MCF-7 [18] and ER-negative MDA-MB231 [17] cell lines. In LNCaP prostate cancer cells, H2A.Z levels are reduced at both promoters and enhancers of the androgen-responsive genes $K L K 3$ and $K L K 2$ genes upon gene activation after androgen treatment [11], while the H2A.Zac/H2A.Z ratio is increased at KLK3 promoter and enhancer regions [20]. Moreover, genome-wide studies in ESCs show that H2A.Zac is enriched at poised and active promoters but not at stably repressed loci [15]. In particular, as transcription activity increases, total H2A.Z decreases at ESC promoters, but an increasing proportion of the variant becomes acetylated [15]. Together these studies suggest that a cross-talk between the total levels of H2A.Z and its acetylation status at gene promoters will dictate the transcriptional fate. Therefore, the lack of analysis of H2A.Z acetylation modification may lead to misconceptions about the actual role of H2A.Z in gene transcription.
H2A.Z is also enriched at enhancer regions [2,21] and notably H2A.Z is reported to be also acetylated at enhancers in ESCs [15,21]. The presence of H2A. $\mathrm{Zac}$ at enhancers opens new avenues for understanding how H2A.Z regulates gene transcription. In fact a recent publication [22] shows that H2A.Z is essential for ER enhancer activity, as it is required for RNA polymerase II ( $\mathrm{Pol}$ II) recruitment, enhancer RNA transcription and enhancer-promoter interactions. Considering the previous studies in breast cancer cell lines where the H2A.Zac/H2A.Z ratio in promoters and enhancers was the determinant factor to induce gene transcription [17,18], it is highly likely that the functions attributed here to H2A.Z in enhancer activity could be caused by the acetylation status. We hypothesize that H2A.Zac is a potential mark of inducible genes, where H2A.Zac nucleosomes provide a more open conformation 'ready' state, which facilitates the binding of transcriptional factors and chromatin remodelers, that is, a 'steady' state, which in turn facilitates Pol II to initiate transcription, 'go' state. However, genome-wide and functional studies focuses on understanding the role of H2A.Zac at both enhancer and promoter regions are needed to further validate this hypothesis.

Even though it is now clear that H2A.Zac is a mark of active transcription, there are only a few studies reporting the functional link and phenotypic consequences of H2A.Z acetylation in the context of gene transcription. A recent study using a non-acetylable H2A.Z mutant investigated the importance of the acetylation in myogenesis. Ectopic expression of the H2A.Z mutant reduced chromatin accessibility to the $\mathrm{MyoD}$ promoter and inhibited $\mathrm{MyoD}$ expression. The lack of this transcription factor produced cell-cycle withdrawal defects, which ultimately resulted in a block of the myogenic differentiation process [16]. This study provides proof of principle that demonstrates the requirement of H2A.Zac is an essential element in the activation of the expression of master regulator genes. Thus, future studies should aim to dissect the specific role and mechanism of the acetylation of $\mathrm{H} 2 \mathrm{~A}$. Z by generating site-specific mutations in normal development and disease.

The underlying molecular mechanism of how acetylation of H2A.Z influences gene activation remains poorly understood. One explanation could be due to changes to the nucleosome structure. The first $(+1)$ nucleosome of the transcription start site is a major barrier to transcription in vivo, and H2A.Z incorporation reduces the nucleosomal barrier to Pol II [23]. In this model, H2A.Z-H2B dimer is more easily lost that the canonical dimer enhances the elongation of Pol II through nucleosomes. Accordingly, H2A.Z shows weaker interactions with $\mathrm{H} 3-\mathrm{H} 4$ compared 
to $\mathrm{H} 2 \mathrm{~A}$ and the acetylation of the $\mathrm{N}$-terminal region of H2A.Z may be responsible for this nucleosome destabilization [8]. On the other hand, histone modifications normally facilitate protein-protein interactions with effector proteins or readers, therefore, the $\mathrm{N}$-terminal of H2A.Z could also represent the target for proteins implicated in transcription. The bromodomain containing transcriptional activator $\mathrm{Brd} 2$ preferentially associates with H2A.Z nucleosomes at active genes, and $\mathrm{Brd} 2$ recruitment is necessary for androgen receptor-regulated gene activation [24]. The fact that bromodomains are recruited to regulatory regions recognizing acetylated lysines, and $\mathrm{H} 2 \mathrm{~A} . \mathrm{Zac}$ is present at androgen-dependant gene promoters [14], it is tempting to hypothesize that H2A.Zac facilitates the recruitment of Brd2 during gene activation. Another recent example of this association occurs in ESCs cells; Surface et al. have proposed a model where Brd2 is absent from bivalent genes enriched for monoubiquitylated H2A.Z, and removal of the ubiquitin group leads to Brd2 recruitment and gene activation of developmental genes [25]. H2A.Zac is present at bivalent and active promoters in ESCs cells and correlates with trancription activity [15], so $\mathrm{Brd} 2$ could be recruited at the promoter and bind to the acetylated lysines of H2A.Z.

\section{References}

1 Zlatanova J, Thakar A. H2A.Z: view from the top. Structure 16(2), 166-179 (2008).

2 Barski A, Cuddapah S, Cui K et al. High-resolution profiling of histone methylations in the human genome. Cell 129(4), 823-837 (2007).

3 Gevry N, Chan HM, Laflamme L, Livingston DM, Gaudreau L. p21 transcription is regulated by differential localization of histone H2A.Z. Genes Dev. 21(15), 1869-1881 (2007).

4 Guillemette B, Gaudreau L. Reuniting the contrasting functions of H2A.Z. Biochem. Cell Biol. 84(4), 528-535 (2006).

5 Weber CM, Henikoff S. Histone variants: dynamic punctuation in transcription. Genes Dev. 28(7), 672-682 (2014).

6 Sevilla A, Binda O. Post-translational modifications of the histone variant H2AZ. Stem Cell Res. 12(1), 289-295 (2014).

7 Millar CB, Xu F, Zhang K, Grunstein M. Acetylation of $\mathrm{H} 2 \mathrm{AZ}$ Lys 14 is associated with genome-wide gene activity in yeast. Genes Dev. 20(6), 711-722 (2006).

8 Ishibashi T, Dryhurst D, Rose KL, Shabanowitz J, Hunt DF, Ausio J. Acetylation of vertebrate H2A.Z and its effect on the structure of the nucleosome. Biochemistry (Mosc). 48(22), 5007-5017 (2009).

9 Binda O, Sevilla A, Leroy G, Lemischka IR, Garcia BA, Richard S. SETD6 monomethylates H2AZ on lysine 7 and is required for the maintenance of embryonic stem cell selfrenewal. Epigenetics 8(2), 177-183 (2013).
This data suggest that $\mathrm{Brd} 2$ could be a specific reader of H2A.Zac to ultimately activate gene transcription.

In conclusion, we purport that the key regulator of transcription is not the present or absence of H2A.Z but its acetylation status. Therefore, studying the incorporation of $\mathrm{H} 2 \mathrm{~A} . \mathrm{Z}$ as a whole without considering the PTMs, is insufficient to understand the mechanism of action of this histone variant. We consider that we have only seen the 'tip of the iceberg' regarding the role of H2A.Z in gene regulation and more detailed studies on the different PTMs are needed to fully understand its complexity.

\section{Financial \& competing interests disclosure}

$F$ Valdes-Mora is a National Breast Cancer Foundation/Cure Cancer Australia Foundation Postdoctoral Training Fellow. SJ Clark is supported by a National Health and Medical Research Council (NHMRC) Fellowship grant (\#1088144). This work is supported by NHMRC project grant (\#1063560). The authors have no other relevant affiliations or financial involvement with any organization or entity with a financial interest in or financial conflict with the subject matter or materials discussed in the manuscript apart from those disclosed.

No writing assistance was utilized in the production of this manuscript.

10 Sarcinella E, Zuzarte PC, Lau PN, Draker R, Cheung P. Monoubiquitylation of H2A.Z distinguishes its association with euchromatin or facultative heterochromatin. Mol. Cell. Biol. 27(18), 6457-6468 (2007).

11 Draker R, Sarcinella E, Cheung P. USP10 deubiquitylates the histone variant $\mathrm{H} 2 \mathrm{~A} . \mathrm{Z}$ and both are required for androgen receptor-mediated gene activation. Nucleic Acids Res. 39(9), 3529-3542 (2011).

12 Halley JE, Kaplan T, Wang AY, Kobor MS, Rine J. Roles for H2A.Z and its acetylation in GAL1 transcription and gene induction, but not GAL1-transcriptional memory. PLoS Biol. 8(6), e1000401 (2010).

13 Bruce K, Myers FA, Mantouvalou E et al. The replacement histone H2A.Z in a hyperacetylated form is a feature of active genes in the chicken. Nucleic Acids Res. 33(17), 5633-5639 (2005).

14 Valdes-Mora F, Song JZ, Statham AL et al. Acetylation of H2A.Z is a key epigenetic modification associated with gene deregulation and epigenetic remodeling in cancer. Genome Res. 22(2), 307-321 (2012).

$15 \mathrm{Ku}$ M, Jaffe JD, Koche RP et al. H2A.Z landscapes and dual modifications in pluripotent and multipotent stem cells underlie complex genome regulatory functions. Genome Biol. 13(10), R85 (2012).

16 Law C, Cheung P. Expression of non-acetylatable H2A.Z in myoblast cells blocks myoblast differentiation through disruption of MyoD expression. J. Biol. Chem. 290 (21), 13234-13249 (2015).

17 Dalvai M, Bellucci L, Fleury L, Lavigne AC, Moutahir F, Bystricky K. H2A.Z-dependent crosstalk between 
enhancer and promoter regulates cyclin D1 expression. Oncogene 32(36), 4243-4251 (2012).

18 Dalvai M, Fleury L, Bellucci L, Kocanova S, Bystricky K. TIP48/Reptin and H2A.Z requirement for initiating chromatin remodeling in estrogen-activated transcription. PLoS Genet. 9(4), e1003387 (2013).

19 Bellucci L, Dalvai M, Kocanova S, Moutahir F, Bystricky K. Activation of $\mathrm{p} 21$ by HDAC inhibitors requires acetylation of H2A.Z. PLoS ONE 8(1), e54102 (2013).

20 Dryhurst D, McMullen B, Fazli L, Rennie PS, Ausio J. Histone H2A.Z prepares the prostate specific antigen (PSA) gene for androgen receptor-mediated transcription and is upregulated in a model of prostate cancer progression. Cancer Lett. 315(1), 38-47 (2012).

21 Hu G, Cui K, Northrup D et al. H2A.Z facilitates access of active and repressive complexes to chromatin in embryonic

\section{Affiliations}

\section{Yolanda Colino-Sanguino}

Histone Variants Group, Genomics \& Epigenetics Division, Garvan Institute of Medical Research, Sydney 2010, New South Wales, Australia

and

Epigenetics Research Laboratory, Genomics \& Epigenetics Division, Garvan Institute of Medical Research, Sydney 2010, New South Wales, Australia

\section{Susan J Clark}

Epigenetics Research Laboratory, Genomics \& Epigenetics Division, Garvan Institute of Medical Research, Sydney 2010, New South Wales, Australia

and

St. Vincent's Clinical School, University of NSW, Sydney 2010,

New South Wales, Australia stem cell self-renewal and differentiation. Cell Stem Cell 12(2), 180-192 (2013).

22 Brunelle M, Nordell Markovits A, Rodrigue S, Lupien M, Jacques PE, Gevry N. The histone variant H2A.Z is an important regulator of enhancer activity. Nucleic Acids Res. 43(20), 9742-9756 (2015).

23 Weber CM, Ramachandran S, Henikoff S. Nucleosomes are context-specific, H2A.Z-modulated barriers to RNA polymerase. Mol. Cell 53(5), 819-830 (2014).

24 Draker R, Ng MK, Sarcinella E, Ignatchenko V, Kislinger T, Cheung P. A combination of H2A.Z and $\mathrm{H} 4$ acetylation recruits $\mathrm{Brd} 2$ to chromatin during transcriptional activation. PLoS Genet. 8(11), e1003047 (2012).

25 Surface LE, Fields PA, Subramanian V et al. H2A.Z.1 monoubiquitylation antagonizes $B R D 2$ to maintain poised chromatin in ESCs. Cell Rep. 14(5), 1142-1155 (2016).

\section{Fatima Valdes-Mora}

Histone Variants Group, Genomics \& Epigenetics Division, Garvan Institute of Medical Research, Sydney 2010, New South Wales, Australia

and Epigenetics Research Laboratory, Genomics \& Epigenetics Division Garvan Institute of Medical Research, Sydney 2010, New South Wales, Australia

and

St. Vincent's Clinical School, University of NSW, Sydney 2010, New South Wales, Australia 